\title{
FAST SOLVERS FOR FINITE DIFFERENCE APPROXIMATIONS FOR THE STOKES AND NAVIER-STOKES EQUATIONS
}

\author{
DONGHO SHIN $^{1}$ and JOHN C. STRIKWERDA ${ }^{2}$
}

(Received 1 December 1994; revised 8 March 1995)

\begin{abstract}
We consider several methods for solving the linear equations arising from finite difference discretizations of the Stokes equations. The two best methods, one presented here for the first time, apparently, and a second, presented by Bramble and Pasciak, are shown to have computational effort that grows slowly with the number of grid points. The methods work with second-order accurate discretizations. Computational results are shown for both the Stokes equations and incompressible Navier-Stokes equations at low Reynolds number.
\end{abstract}

\section{Introduction}

The steady-state Stokes equations in $R^{d}$ are

$$
\nabla^{2} \vec{u}-\vec{\nabla} p=\vec{f}, \quad \vec{\nabla} \cdot \vec{u}=g, \quad \text { in } \Omega \subset R^{d} .
$$

In almost all applications the function $g$ in the second equation of (1.1) is zero, but the methods discussed here do not require that $g$ be zero, so we include this slightly more general case. We consider only the Dirichlet boundary condition, $\vec{u}=\vec{b}$ on $\partial \Omega$. The methods discussed here should be easy to extend to other boundary conditions. The velocity $\vec{u}$ is a vector of dimension $d$ and the pressure $p$ is a scalar. The domain of our computational examples is the unit square in $R^{2}$. In current research we are using these methods on more general domains.

Let $A_{h}, G_{h}$ and $D_{h}$ be the matrices generated by discretizations of the differential operators $\left(-\nabla^{2}\right), \vec{\nabla}$ and $(-\vec{\nabla} \cdot)$, respectively. The discretization of (1.1) may be written as

$$
\left(\begin{array}{cc}
A_{h} & G_{h} \\
D_{h} & 0
\end{array}\right)\left(\begin{array}{c}
u_{h} \\
p_{h}
\end{array}\right)=\left(\begin{array}{l}
f_{h} \\
g_{h}
\end{array}\right)
$$

\footnotetext{
${ }^{1}$ Department of Mathematics, Inje University, Kimhae, Kyungnam 612-749, Korea

${ }^{2}$ Department of Computer Sciences and Center for the Mathematical Sciences, University of WisconsinMadison, Madison, WI 49506 U.S.A.
}

(C) Australian Mathematical Society, 1996, Serial-fee code 0334-2700/96 
In some formulations of the difference equations, for example, staggered grids and finite element formulations, the matrix $D_{h}$ is the transpose of $G_{h}$, that is, $D_{h}=G_{h}^{\top}$. However, in many cases this is not true, either because of boundary conditions or because of the difference schemes. In this paper we do not assume that $D_{h}=G_{h}^{\top}$. Note that

$$
\operatorname{det}\left(\begin{array}{cc}
A_{h} & G_{h} \\
D_{h} & 0
\end{array}\right)=\operatorname{det}\left(A_{h}\right) \operatorname{det}\left(0-D_{h} A_{h}^{-1} G_{h}\right) .
$$

Hence (1.2) is solvable if $A_{h}$ and $Q_{h}$ are invertible, where

$$
Q_{h}=D_{h} A_{h}^{-1} G_{h} .
$$

The methods we discuss here are based on the operator $Q_{h}$. We note that

$$
u_{h}=A_{h}^{-1}\left(f_{h}-G_{h} p_{h}\right)
$$

from the first row in (1.2). Using the second row, we have

$$
D_{h} A_{h}^{-1}\left(f_{h}-G_{h} p_{h}\right)=g_{h} .
$$

Thus (1.2) can be solved by first solving

$$
Q_{h} p_{h}=h_{h}
$$

for $p_{h}$ where

$$
h_{h}=D_{h} A_{h}^{-1} f_{h}-g_{h} .
$$

After $p_{h}$ is obtained, $u_{h}$ can be recovered from (1.3). The operator $Q_{h}$ is the Schur complement of the system (1.2).

The operator $Q_{h}$ often has several rather desirable properties. As we show in the next section, $Q_{h}$ is close to being a symmetric, positive definite operator. Moreover, in many cases the eigenvalues of $Q_{h}$ can be bounded independently of the mesh spacing. In this case, one can use the conjugate gradient method to solve (1.4), and the number of conjugate gradient iterations required to solve (1.4) should be relatively independent of the grid parameters. We call the iterative method based on solving (1.4) by the conjugate gradient method the pressure equation method, and refer to it as the PE method.

The PE method requires that $A_{h}$ needs to be inverted in each iteration of the conjugate gradient method. This must be done efficiently in order for the overall method to be efficient. Multigrid methods or preconditioned conjugate gradient methods are two possible methods. The price for inversion of $A_{h}$ would be essentially independent of the grid size when the multigrid method is used, and would grow slowly if a preconditioned conjugate gradient method were used. 
The Uzawa iterative method, see [1], can be viewed as solving (1.4) by a fixed point iteration. This method can be written as,

$$
\begin{aligned}
A_{h} u_{h}^{\nu+1} & =-G_{h} p_{h}^{\nu}+f_{h} \\
p_{h}^{\nu+1} & =p_{h}^{\nu}-\gamma\left(D_{h} u_{h}^{\nu+1}-g_{h}\right) .
\end{aligned}
$$

The method converges for $\gamma$ in some interval $(0, \bar{\gamma})$ depending on the scaling of the operators.

A potential disadvantage of these methods is the necessity of inverting $A_{h}$ at each iteration. There have been a number of iterative methods that avoid the inversion of the operator $A_{h}$ as required by the Uzawa method. We describe only a few here. For other methods see [1], and [8].

Bramble and Pasciak [3] proposed an iterative method using a preconditioned conjugate gradient method to solve finite element approximations to the Stokes equations. To avoid the inversion of $A_{h}$, Bramble and Pasciak used a preconditioner $A_{h 0}^{-1}$. With the preconditioner, (1.2) is transformed to

$$
M_{h}\left(\begin{array}{c}
u_{h} \\
p_{h}
\end{array}\right)=\hat{f}_{h}
$$

where

$$
M_{h}=\left(\begin{array}{cc}
A_{h 0}^{-1} A_{h} & A_{h 0}^{-1} G_{h} \\
D_{h} A_{h 0}^{-1}\left(A_{h}-A_{h 0}\right) & D_{h} A_{h 0}^{-1} G_{h}
\end{array}\right) \text { and } \hat{f}_{h}=\left(\begin{array}{c}
A_{h 0}^{-1} f_{h} \\
D_{h} A_{h 0}^{-1} F_{h}-g_{h}
\end{array}\right) .
$$

They assumed that $G_{h}^{*}=D_{h}$ and

$$
0<\left(\left(A_{h}-A_{h 0}\right) u_{h}, u_{h}\right) \leq \alpha\left(A_{h} u_{h}, u_{h}\right)
$$

for all $u_{h} \neq 0$ and for some $\alpha$ with $0<\alpha<1$. If (1.7) is satisfied, the $M_{h}$ is symmetric and positive definite with the inner product

$$
\left.\left[\left(\begin{array}{c}
u_{h} \\
p_{h}
\end{array}\right),\left(\begin{array}{l}
v_{h} \\
q_{h}
\end{array}\right)\right]=\left(A_{h} u_{h}, v_{h}\right)-A_{h 0} u_{h}, v_{h}\right)+\left(p_{h}, q_{h}\right)
$$

where $(\cdot, \cdot)$ is the usual inner product in the discrete space.

Under an assumption equivalent to the inf-sup condition (see [2]) which implies that the condition number $\kappa\left(M_{h}\right)$ of $M_{h}$ is bounded by a constant independent of $h$, they showed that

$$
0<C_{1}\left\|\left(\begin{array}{c}
u_{h} \\
p_{h}
\end{array}\right)\right\|^{2} \leq\left[M_{h}\left(\begin{array}{c}
u_{h} \\
p_{h}
\end{array}\right),\left(\begin{array}{c}
u_{h} \\
p_{h}
\end{array}\right)\right] \leq C_{2}\left\|\left(\begin{array}{c}
u_{h} \\
p_{h}
\end{array}\right)\right\|^{2}
$$


for some positive constants $C_{1}$ and $C_{2}$ and for all $\left(u_{h}, p_{h}\right)^{\top}$. This implies that $\kappa\left(M_{h}\right)$ is bounded by a constant independent of $h$ and the conjugate gradient is a good method to solve (1.6). We refer to the above iterative method suggested by Bramble and Pasciak as the BP method.

Strikwerda [12] avoided the inversion of $A_{h}$ by using one step of successive-overrelaxation. If $A_{h}$ is written as

$$
A_{h}=\Delta_{h}-L_{h}-U_{h}
$$

where $\Delta_{h}$ is the diagonal of $A_{h}$ and $L_{h}$ and $U_{h}$ are strictly lower and upper triangular matrices respectively, then the method introduced in [12] is

$$
\begin{aligned}
& u_{h}^{v+1}=u_{h}^{v}-\omega \Delta_{h}^{-1}\left(\Delta_{h} u_{h}^{v}-L_{h} u_{h}^{v+1}-U_{h} u_{h}^{v}+G_{h} p_{h}^{v}-f_{h}\right) \\
& p_{h}^{v+1}=p_{h}^{v}-\gamma\left(D_{h} u_{h}^{v+1}-g_{h}\right) .
\end{aligned}
$$

We refer to this method as the SOR method.

The number of iterations required by the SOR method is, at best, proportional to $h^{-1}$ where $h$ denotes mesh size, and this requires a great amount of time to get a solution for small mesh sizes. For example, Strikweda and Scarbnick [14] pointed out that the SOR method was quite slow when they used domain decomposition methods. An advantage of the SOR method is the relative simplicity of coding the algorithm.

The PE method is the fastest of the methods we compare here. Both the PE and the BP method have work that is proportional to the number of grid points, but the PE method is faster. In part this is because the PE method needs to invert $A_{h}$ just once in each conjugate gradient step, while the BP method needs to operate with $A_{h 0}^{-1}$ twice. The other reason is that the inner product used in the BP method requires considerable work to compute. This extra work cancels out the advantage of using the preconditioner. The exact comparison of efficiency is performed in Section 4.

The PE method does not require parameters. On the other hand, the SOR method needs some effort to find good values of the parameters $\omega$ and $\gamma$. The BP method also needs a scaling parameter in the preconditioning and, in our experiments, the method was very sensitive to the scaling parameter. In the two subsequent sections, we discuss the PE method and the BP method.

\section{Analysis of the PE method}

To analyze the PE method, we first examine the analogous problem for the partial differential equations. Define the operator $Q$ for $p$ in $L^{2}(\Omega) / R$ as

$$
Q p:=\vec{\nabla} \cdot \vec{\psi}
$$


where

$$
\nabla^{2} \vec{\psi}=\vec{\nabla} p \quad \text { with }\left.\quad \vec{\psi}\right|_{\partial \Omega}=0 .
$$

$Q$ can be expressed symbolically as $(-\vec{\nabla} \cdot)\left(-\nabla^{2}\right)^{-1}(\vec{\nabla})$. Crozier [7] has proved the following theorem, see also [9].

THEOREM 2.1. If $\Omega$ is a connected, bounded domain in $R^{2}$ with smooth boundary, then the operator $Q$ is a bounded, positive-definite operator, with bounded inverse, on $L^{2}(\Omega) / R$.

The norm of $Q$ is actually bounded by 1 . So the above theorem can be expressed mathematically as

$$
0<C\|p\|^{2} \leq(Q p, p) \leq\|p\|^{2}
$$

for some positive constant $C$ and for all $p$. Moreover the operator $Q$ is self-adjoint.

Even more can be said about the eigenvalues of $Q$. The eigenvalue 1 occurs with infinite multiplicity. This is on the orthogonal compliment of the harmonic functions in $L^{2}(\Omega)$. We conjecture, based on some evidence, that the rest of the eigenvalues are clustered around one-half.

CONJECTURE. The operator $Q$ has the eigenvalue 1 with an infinite multiplicity, and the remaining eigenvalues have a cluster point at $1 / 2$ with no other cluster point.

If $Q_{h}$ is a consistent and regular finite difference approximation to $Q$, then one can expect that $Q_{h}$ is positive-definite and has its condition number bounded by a constant independent of $h$.

If one uses the usual central difference scheme for $D_{h}$ and $G_{h}$, then $Q_{h}$ is symmetric. However, if central difference formulas are used for $D_{h}$ and $G_{h}$ then the scheme is not regular, see [11], and $Q_{h}$ will either be singular or be nearly singular.

If the regularized central difference scheme (see [11]) is used for $D_{h}$ and $G_{h}$, then the symmetry of $Q_{h}$ is lost. However $Q_{h}$ is close to being symmetric. As our numerical solutions show, the ordinary conjugate gradient method, applied to $Q_{h}$ discretized by this scheme, works very well.

The following is the conjugate gradient method we used to find the pressure $p_{h}$, see [13]. Let $\left(u_{h}^{0}, p_{h}^{0}\right)$ be an initial solution with $u_{h}^{0}$ having the true boundary values. Let

$$
s_{h}^{0}=r_{h}^{0}=h_{h}-Q_{h} p_{h}^{0}
$$

where $s_{h}$ and $r_{h}$ denote the search vector and residual vectors, respectively. Define

$$
q_{h}^{0}=Q_{h} r_{h}^{0}
$$


The conjugate gradient method for the PE method is

$$
\begin{aligned}
p_{h}^{v+1} & =p_{h}^{v}+\alpha_{v} s_{h}^{v} \\
r_{h}^{v+1} & =r_{h}^{\nu}-\alpha_{\nu} q_{h}^{v} \\
s_{h}^{v+1} & =r_{h}^{v+1}+\beta_{\nu} q_{h}^{v} \\
q_{h}^{v+1} & =Q_{h} r_{h}^{\nu+1}+\beta_{\nu} q_{h}^{v} \\
\alpha_{n} u & =\left(r_{h}^{v}, r_{h}^{\nu}\right) /\left(s_{h}^{\nu}, q_{h}^{\nu}\right) \\
\beta_{v} & =\left(r_{h}^{\nu+1}, r_{h}^{\nu+1}\right) /\left(r_{h}^{v}, r_{h}^{\nu}\right) .
\end{aligned}
$$

When $A_{h}$ is inverted, the boundary values must be assigned to obtain a unique solution. The residual vector $r_{h}$ in the conjugate gradient method is defined to be $h_{h}-Q_{h} p_{h}$ and initially $r_{h}^{0}=D_{h} A_{h}^{-1}\left(f_{h}-G_{h} p_{h}^{0}\right)-g_{h}$. The first row in (1.2) implies that the boundary values of $A_{h}^{-1}\left(f_{h}-G_{h} p_{h}^{0}\right)$ have to be the boundary values of $u_{h}$, the velocity field of the solution. But, in later steps, when one needs to evaluate $Q_{h} r_{h}$, the zero boundary values for $A_{h}^{-1}$ should be used, since the residual vector converges to zero as the iteration number grows.

The multigrid process using $V$-cycles was used to invert $A_{h}$. The ordinary GaussSeidel iteration was used as the smoother. The number of relaxations in each node of the multigrid was 2 . Injection was used to go to a coarser level and interpolation was used to go to a finer level. The residual was computed just before the injection process and at the end of the $V$-cycles. For the multigrid terminology, refer to [6].

\section{BP method}

The conjugate gradient method applied to (1.6) is defined as the following, refer to [3] for details. Let

$$
z_{h}^{0}=\left(u_{h}^{0}, p_{h}^{0}\right)^{t}
$$

be an initial approximation to the solution with the true boundary values assigned for $u_{h}^{0}$. With

$$
s_{h}^{0}=r_{h}^{0}=\hat{f}_{h}-M_{h} z_{h}^{0},
$$

define for $v \geq 0$

$$
\begin{aligned}
\alpha_{\nu} & =\frac{\left[r_{h}^{v}, s_{h}^{\nu}\right]}{\left[M_{h} s_{h}^{v}, s_{h}^{\nu}\right]}, \\
z_{h}^{v+1} & =z_{h}^{v}+\alpha_{\nu} s_{h}^{\nu}, \\
r_{h}^{v+1} & =\hat{f}_{h}-M_{h} z_{h}^{\nu+1},
\end{aligned}
$$




$$
\begin{aligned}
\beta_{v} & =-\frac{\left[M_{h} s_{h}^{\nu}, r_{h}^{\nu+1}\right]}{\left[M_{h} s_{h}^{\nu}, s_{h}^{\nu}\right]}, \\
s_{h}^{\nu+1} & =r_{h}^{\nu+1}+\beta_{\nu} s_{h}^{v} .
\end{aligned}
$$

Note that, from [13],

$$
\alpha_{\nu}=\frac{\left[r_{h}^{\nu}, r_{h}^{\nu}\right]}{\left[M_{h} s_{h}^{\nu}, s_{h}^{\nu}\right]} \quad \text { and } \quad \beta_{v}=\frac{\left[r_{h}^{\nu+1}, r_{h}^{\nu+1}\right]}{\left[r_{h}^{\nu}, r_{h}^{\nu}\right]}
$$

Since $M_{h}$ is positive-definite, (3.2) shows that $\alpha_{v}$ and $\beta_{v}$ are nonnegative. This fact can be used to test a good candidate for $A_{h 0}^{-1}$. One possible choice for $A_{h 0}^{-1}$ is to let it be one $V$-cycle for solving

$$
A_{h} u_{h}-f_{h}
$$

when the boundary values of $u_{h}$ are specified. However this choice of $A_{h 0}^{-1}$ may not satisfy (1.7). A better choice is to take

$$
A_{h 0}^{-1}=\sigma A_{h 1}^{-1}
$$

where $A_{h 1}^{-1}$ is one $V$-cycle for solving (3.3) and $\sigma$ is a scaling factor. If $\sigma$ is chosen improperly, then there is a chance for $M_{h}$ to be indefinite. This is detected in computation by checking on the positivity of $\alpha_{\nu}$ and $\beta_{\nu}$. By changing the value of $\sigma$, one is able to find a $A_{h 0}^{-1}$ satisfying (1.7).

The parameter $\sigma$ is not hard to find since it is larger than and close to 1 by the following argument. Since $A_{h 1}^{-1} A_{h} \approx I_{h}$, one has $\sigma A_{h 1}^{-1} A_{h} \approx I_{h}$ also for $\sigma$ near 1 . Note that $A_{h}-A_{h 1} \approx 0$. To get $A_{h}-\sigma^{-1} A_{h 1}>0, \sigma$ needs to be larger than and close to 1 .

The following comments explain how we implemented the BP method. Some care must be taken to insure good efficiency. From (1.6) and the definition of $M_{h}$, the residual vector is

$$
r_{h}=\left(\begin{array}{c}
A_{h 0}^{-1}\left(f_{h}-A_{h} u_{h}-G_{h} p_{h}\right) \\
D_{h} A_{h 0}^{-1}\left(f_{h}-A_{h} u_{h}-G_{h} p_{h}\right)+D_{h} u_{h}-g_{h}
\end{array}\right) .
$$

To compute $r_{h}$, first set and save the vector

$$
w_{h}:=f_{h}-A_{h} u_{h}-G_{h} p_{h}
$$

for later use. Next the system

$$
A_{h 0} \hat{w}_{h}=w_{h}
$$


is solved for $\hat{w}_{h}$ with zero boundary condition, and

$$
r_{h}=\left(\begin{array}{c}
r_{l} \\
r_{I I}
\end{array}\right):=\left(\begin{array}{c}
\hat{w}_{h} \\
D_{h}\left(\hat{w}_{h}+u_{h}\right)-g_{h}
\end{array}\right) .
$$

In this way the initial residual $r_{h}^{0}$ is computed. Also set $s_{h}^{0}=r_{h}^{0}$.

In subsequent iterations, the inner product $\left[r_{h}, s_{h}\right]$ is computed as

$$
\begin{aligned}
{\left[r_{h}, s_{h}\right] } & =\left(\left(A_{h}-A_{h 0}\right) r_{I}, s_{I}\right)+\left(r_{I I}, S_{I I}\right) \\
& =\left(A_{h} r_{I}-w_{h}, s_{I}\right)+\left(r_{I I}, s_{I I}\right)
\end{aligned}
$$

where $s_{h}=\left(S_{l}, S_{H I}\right)^{\top}$. The last expression is used to compute $\left[r_{h}, s_{h}\right]$. Note that $A_{h 0}$ is not used explicitly. To compute $\left[M_{h} s_{h}, s_{h}\right]$, note that

$$
\begin{aligned}
{\left[M_{h}\left(\begin{array}{c}
s_{I} \\
s_{I I}
\end{array}\right),\left(\begin{array}{c}
s_{I} \\
S_{I I}
\end{array}\right)\right]=} & \left(A_{h} A_{h 0}^{-1}\left(A_{h} s_{I}+G_{h} S_{I I}\right)-\left(A_{h} s_{I}+G_{h} s_{I I}\right), s_{I}\right) \\
& \left.+D_{h} A_{h 0}^{-1}\left(A_{h}-A_{h 0}\right) s_{I}+D_{h} A_{h 0}^{-1} G_{h} S_{I I}, S_{I I}\right)
\end{aligned}
$$

To simplify this expression, set

$$
w_{h}:=A_{h} s_{I}+G_{h} S_{I I}
$$

and solve

$$
A_{h 0} \hat{w}_{h}=w_{h}
$$

for $\hat{w}_{h}$ with zero boundary condition. If

$$
m_{l}:=A_{h} \hat{w}_{h}-w_{h}, \quad m_{I l}:=D_{h}\left(\hat{w}_{h}-s_{l}\right)
$$

then

$$
\left[M_{h}\left(\begin{array}{c}
s_{I} \\
S_{I I}
\end{array}\right),\left(\begin{array}{c}
s_{I} \\
S_{I I}
\end{array}\right)\right]=\left(m_{I}, s_{I}\right)+\left(m_{I I}, S_{I I}\right)
$$

If the vector $\left(m_{l}, m_{l l}\right)^{\top}$ is saved, then $\left[M_{h} s_{h}, r_{h}\right]$ is computed as

$$
\left(m_{l}, r_{l}\right)+\left(m_{l l}, r_{I I}\right)
$$

In this whole process, we need to evaluate $A_{h 0}^{-1}$, and never need to evaluate $A_{h 0}$ itself, and this is necessary since a multigrid process is used to define $A_{h 0}^{-1}$. The special forms of $\alpha_{v}$ and $\beta_{v}$ in (3.1) were chosen to be easily computable. 


\section{Analysis of efficiency}

In this section the total number of significant operations are estimated, which we designate as TSO, for each iterative method. These estimates are used to compare the efficiency of each of these methods. We take as a representative case the Stokes equations on a square in $R^{2}$ or cube in $R^{3}$. If $N+1$ is the number of grid points in a coordinate direction in $R^{d}$, then $(N-1)^{d}$ is the number of interior grid points. $\mathrm{TSO}_{S}, \mathrm{TSO}_{P}$ and $\mathrm{TSO}_{B}$ are the TSO for the SOR method, the PE method, and the BP method, respectively. Iter $S$, Iter $P$, and $\operatorname{Iter}_{B}$ are defined similarly.

Let $N_{A}, N_{G}$, and $N_{D}$ be the number of multiplications per grid point to apply $A_{h}$, $G_{h}$ and $D_{h}$, respectively. If $u_{h}=\left(u_{1}, \ldots, u_{d}\right)^{\top}$, then

$$
\left(A_{h} u_{h}\right)_{l, m}=\left(\left(\nabla_{h}^{2} u_{1}\right)_{l, m}, \ldots,\left(\nabla_{h}^{2} u_{d}\right)_{l, m}\right)^{\top} .
$$

We used the usual second-order accurate discrete Laplacian for $\nabla_{h}^{2}$. Since $A_{h}$ involves $d$ scalar Laplacians, $N_{A} \approx(2 d+1) d$. The regularized central differencing was used to find any first derivative with respect to any direction, and this requires 4 points to evaluate. Each of $\left(G_{h} p_{h}\right)_{l, m}$ and $\left(D_{h} u_{h}\right)_{l, m}$ requires $d$ first derivatives to be evaluated, so $N_{G} \approx 4 d$ and $N_{D} \approx 4 d$. We consider our "cost" to be the number of multiplications required.

LEMMA 4.1. $\mathrm{TSO}_{S} \approx \mathrm{Iter}_{S} \cdot d(2 d+9) \cdot(N-1)^{d}$.

PROOF. From (1.9),

$$
\begin{aligned}
\operatorname{TSO}_{S} & \approx \operatorname{Iter}_{S} \cdot\left(N_{A}+N_{G}+N_{D}\right) \cdot(N-1)^{d} \\
& \approx \operatorname{Iter}_{S} \cdot\left(2 d^{2}+d+8 d\right) \cdot(N-1)^{d} \\
& \approx \operatorname{Iter}_{S} \cdot\left(2 d^{2}+9 d\right) \cdot(N-1)^{d}
\end{aligned}
$$

LEMMA 4.2. One $V$-cycle for the scalar second-order Laplacian costs approximately $N_{V}(N-1)^{d}$ where $N_{V}=2^{d}\left(2^{d}-1\right)^{-1}(10 d+6)$.

Proof. Going down along a $V$-cycle, we do 2 smoothing processes, 1 residual finding, and 1 injection at each level. On the way up, we do 2 smoothing processes and 1 interpolation at each level. So, in a $V$-cycle, altogether 4 smoothing processes, 1 residual finding, 1 injection and 1 interpolation at each level are needed. On the finest level, smoothing costs $(2 d+1)(N-1)^{d}$, computing the residual is about the same, and injection and interpolation together cost at most $(N-1)^{d}$ operations.

Thus one $V$-cycle costs roughly

$$
(5(2 d+1)+1) \cdot(N-1)^{d}\left(1+\frac{1}{2^{d}}+\left(\frac{1}{2^{d}}\right)^{d}+\cdots+\left(\frac{1}{2^{d}}\right)^{\# \text { of levels }}\right)
$$


where $d$ is the dimension of our domain. The above number is approximately $2^{d} /\left(2^{d}-\right.$ 1) $(10 d+6)(N-1)^{d}$.

LEMMA 4.3. $\mathrm{TSO}_{P} \approx \operatorname{Iter}_{P} \cdot d\left(8+\bar{v} N_{V}\right) \cdot(N-1)^{d}$, where $\bar{v}$ is the average number of $V$-cycles required per iteration.

PROOF. One needs to apply the matrix $Q_{h}$ in each conjugate gradient iteration. From (4.1), we see that $A_{h}^{-1}$ consists of $d$ multigrid operations. So, we have by Lemma 4.2

$$
\begin{aligned}
\operatorname{TSO}_{P} & \approx \operatorname{Iter}_{P} \cdot\left(N_{G}+N_{D}+d \bar{v} \cdot N_{V}\right) \cdot(N-1)^{d} \\
& \approx \operatorname{Iter}_{P} \cdot\left(8 d+d \bar{v} N_{V}\right) \cdot(N-1)^{d} .
\end{aligned}
$$

LEMMA 4.4. $\mathrm{TSO}_{B} \approx \operatorname{Iter}_{B} \cdot 2 d\left(4 d+10+N_{V}\right) \cdot(N-1)^{d}$.

PROOF. In each iteration, the main effort is in finding $r_{h},\left[r_{h}, s_{h}\right]$ and $\left[M_{h} s_{h}, s_{h}\right]$ from (3.1). By Lemma 4.2 and the equations from (3.4) to (3.6), the cost to get $r_{h}$ is

$$
\left(N_{A}+N_{G}+d \cdot N_{V}+N_{D}\right)(N-1)^{d} \text {. }
$$

Evaluating $\left[r_{h}, s_{h}\right]$ costs

$$
N_{A} \cdot(N-1)^{d}
$$

by (3.7).

The cost of evaluating $\left[M_{h} s_{h}, s_{h}\right]$ is

$$
\left(2 N_{A}+N_{G}+d N_{V}+N_{D}\right)(N-1)^{d}
$$

by the equations from (3.8) to (1.6).

Adding these costs, we obtain

$$
\begin{aligned}
\mathrm{TSO}_{B} & \approx \operatorname{Iter}_{B} \cdot\left(4 N_{A}+2 N_{G}+2 N_{D}+2 d N_{V}\right) \cdot(N-1)^{d} \\
& \approx \operatorname{Iter}_{B} \cdot\left(8 d^{2}+4 d+16 d+2 d N_{V}\right) \cdot(N-1)^{d} \\
& \approx \operatorname{Iter}_{B} \cdot\left(8 d^{2}+20 d+2 d N_{V}\right) \cdot(N-1)^{d} .
\end{aligned}
$$

By (1.8) and (2.1), Iter $P$ and Iter $_{B}$ are bounded by some constants not depending on mesh size. Moreover, Iter ${ }_{S}$ is proportional to $N$ at best. For the test case considered in Section 6 we find, for $N=64$ and $d=2$, Iter ${ }_{S} \approx 8(N-1)$, $\operatorname{Iter}_{P}=12$, and Iter $_{B}=17$. Also, $\bar{v}$ was about 2 for the PE method. So, $\mathrm{TSO}_{S} \approx 208(N-1)^{3}$, $\mathrm{TSO}_{P} \approx 1856(N-1)^{2}$, and $\mathrm{TSO}_{B} \approx 3581(N-1)^{3}$.

We see that the PE is the fastest method, with the BP method being about twice as much work. The SOR method is 7 times as much work as the PE method for the test case considered here and is even less efficient as $N$ increases. The numerical results in Section 6 also show that based on CPU time, for this test case, the PE method is more than 7 times faster than the SOR method and about two times faster than the BP method, agreeing with our analysis. 


\section{The numerical experiments}

For the numerical experiment, we used the Stokes equations of the form

$$
\begin{aligned}
& \nabla^{2} u-\frac{\partial p}{\partial x}=-2 \pi^{2} \sin \pi x \sin \pi y+\pi \sin \pi x \sin \pi y, \\
& \nabla^{2} u-\frac{\partial p}{\partial x}=-2 \pi^{2} \cos \pi x \cos \pi y-\pi \cos \pi x \cos \pi y, \\
& \frac{\partial u}{\partial x}+\frac{\partial v}{\partial y}=0,
\end{aligned}
$$

on $0<x, y<1$ with $u$ and $v$ specified on the boundary.

The exact solution is given by

$$
u=\sin \pi x \sin \pi y, \quad v=\cos \pi x \cos \pi y, \quad p=\cos \pi x \sin \pi y .
$$

The discretization used a uniform grid with the same number of grid points in each direction. The second order accurate 5-point Laplacian was used to approximate $\nabla^{2}$ for the above iterative methods.

We employed, for all the iterative methods, the regularized central difference (see [11]) given by

$$
\begin{aligned}
& \frac{\partial p}{\partial x} \approx \delta_{x 0} p_{h}-\frac{h^{2}}{6} \delta_{x-} \delta_{x+}^{2} p_{h}, \\
& \frac{\partial p}{\partial y} \approx \delta_{y 0} p_{h}-\frac{h^{2}}{6} \delta_{y-} \delta_{y+}^{2} p_{h}, \\
& \frac{\partial u}{\partial x} \approx \delta_{x 0} u_{h}-\frac{h^{2}}{6} \delta_{x+} \delta_{x-}^{2} u_{h}, \\
& \frac{\partial v}{\partial y} \approx \delta_{y 0} v_{h}-\frac{h^{2}}{6} \delta_{y+} \delta_{y-}^{2} v_{h},
\end{aligned}
$$

where $h$ is the grid spacing and $\delta_{x 0}, \delta_{x+}$ and $\delta_{x-}$ are the centered, forward, and backward difference operators in the $x$-direction. The operators $\delta_{y 0}, \delta_{y+}$ and $\delta_{y-}$ are defined similarly for the $y$-direction.

To obtain the pressure on the boundary, we used the quadratic interpolation, for example,

$$
p_{0 m}=2 p_{1 m}-p_{2 m},
$$

for all the iterative methods.

The SOR method was stopped when the quantities

$$
\left\|u_{h}^{n+1}-u_{h}^{n}\right\|, \quad\left\|v_{h}^{n+1}-v_{h}^{n}\right\|, \quad\left\|p_{h}^{n+1}-p_{h}^{n}\right\|
$$


were all less than $5 \times 10^{-5}, 10^{-4}$ and $2 \times 10^{-4}$ for mesh sizes $1 / 32,1 / 48$ and $1 / 64$ respectively. These values were chosen because the quantities in (5.1) could not be made much smaller than these values. We did not investigate why these quantities could not be made smaller. The norms of $u_{h}$ and $v_{h}$ in (5.1) were the discrete $L^{2}$ norms, and the norm for $p_{h}$ was the $L^{2}$ norm in its quotient space (see [12]). The relaxation parameters $\omega$ and $\gamma$ were given by

$$
\omega=2 /\left(1+c_{0} h\right), \quad \gamma=c_{1} h
$$

where $c_{0}=3.14$ and $c_{1}=4.5$. See [11] and [12] for more details.

The PE method was stopped when the residual was less that $10^{-6}$. In each conjugate gradient iteration of the PE method, the multigrid process using $V$-cycles was used to invert $A_{h}$. We found that to achieve good overall accuracy it was only necessary to do enough $V$-cycles to reduce the residual in the $L^{2}$ norm to less than $10^{-4}$. Each multigrid process to solve $A_{h} u_{h}=f_{h}$ for $u_{h}$ was stopped when either the number of $V$-cycles was 4 or the residual error was less than $10^{-4}$. The maximum number of $V$-cycles was chosen to be 4 since the residual error didn't change significantly after $4 V$-cycles. Because the reduction factor of the error is small in the multigrid process, more than $4 V$-cycles would rarely be needed. With these stopping criteria, the average number of $V$-cycles needed in each conjugate gradient iteration was 2 .

The BP method was stopped when the residuals were less than $3 \times 10^{-4}, 10^{-4}$ and $3 \times 10^{-5}$ for mesh sizes $1 / 32,1 / 48$ and $1 / 64$ respectively. These values were chosen since, similar to the SOR method, the residuals decreased to values slightly smaller than these values, but could not be made much smaller. In the BP method, several values were run for $\sigma$ and the value of 1.2 worked well.

\section{Test results}

Tables 1, 2, and 3 show the errors for the PE method, the BP method and the SOR method. The column labeled "time" shows the CPU time required for the total computation.

TABLE 1. Errors and CPU time for the PE method.

\begin{tabular}{c|cccc}
\hline$N$ & iter & $u$ & $p$ & time \\
\hline 32 & 12 & $6.46(-5)$ & $2.71(-3)$ & 1.617 \\
48 & 12 & $2.35(-5)$ & $1.25(-3)$ & 4.347 \\
64 & 12 & $1.38(-5)$ & $6.91(-4)$ & 8.362 \\
\hline
\end{tabular}


TABLE 2. Errors and CPU time for the BP method.

\begin{tabular}{c|cccc}
\hline$N$ & iter & $u$ & $p$ & time \\
\hline 32 & 14 & $6.34(-5)$ & $3.04(-3)$ & 2.843 \\
48 & 16 & $2.19(-5)$ & $1.36(-3)$ & 8.558 \\
64 & 17 & $1.19(-5)$ & $7.83(-4)$ & 17.162 \\
\hline
\end{tabular}

TABLE 3. Errors and CPU time for the SOR method.

\begin{tabular}{c|cccc}
\hline$N$ & iter & $u$ & $p$ & time \\
\hline 32 & 275 & $6.33(-5)$ & $2.74(-3)$ & 8.546 \\
48 & 399 & $2.48(-5)$ & $1.26(-3)$ & 28.150 \\
64 & 511 & $1.39(-5)$ & $6.98(-4)$ & 65.214 \\
\hline
\end{tabular}

By comparing CPU times, one can see that the PE method is most efficient, the BP method takes about twice as much effort, and the SOR method is least efficient, taking about 7 times as much time as the PE method. Note that the number of iterations taken by the PE method and the BP method are essentially independent of mesh size, which supports (1.7) and (2.1).

The next table (Table 4) shows the accuracy of the PE method, the BP method, and the SOR method. The order of accuracy was obtained from the formula

$$
\log \left(\operatorname{error}\left(h_{2}\right) / \text { error }\left(h_{1}\right)\right) / \log \left(h_{2} / h_{1}\right)
$$

where $h_{1}, h_{2}$ are mesh sizes. All numerical solutions show that they are second order accurate.

TABLE 4. Order of accuracy for the computed solutions.

\begin{tabular}{c|cc|cc|cc}
\hline \multirow{2}{*}{$N_{1}, N_{2}$} & \multicolumn{2}{|c|}{ PE } & \multicolumn{2}{c|}{ BP } & \multicolumn{2}{c}{ SOR } \\
\cline { 2 - 7 } & $u$ & $p$ & $u$ & $p$ & $u$ & $p$ \\
\hline 64,48 & 2.1 & 2.0 & 1.9 & 2.1 & 2.1 & 1.9 \\
64,32 & 2.2 & 2.0 & 2.2 & 2.0 & 2.4 & 2.0 \\
48,32 & 2.3 & 1.9 & 2.5 & 1.9 & 2.6 & 2.0 \\
\hline
\end{tabular}




\section{Navier-Stokes equations}

The steady-state Navier-Stokes equations in $R^{d}$ are of the form

$$
\begin{aligned}
-R^{-1} \nabla^{2} \vec{u}+(\vec{u} \cdot \vec{\nabla}) \vec{u}+\vec{\nabla} p & =\vec{f}, \\
\vec{\nabla} \cdot \vec{u} & =g \quad \text { in } \Omega \subset R^{d}
\end{aligned}
$$

where $R$ is the Reynolds number. We consider the Dirichlet boundary condition

$$
\vec{u}=\vec{b} \quad \text { on } \partial \Omega \text {. }
$$

There are several possible extensions of the PE method from the Stokes equations to the Navier-Stokes equations, depending on how one linearizes the first equation in (7.1). To apply the PE method efficiently to (7.1), we used the following algorithm which worked for $R$ up to about 100 .

(1) Start with an initial solution $\vec{u}^{0}, p^{0}$.

(2) Given the solution $\vec{u}^{\nu}$, let

$$
\vec{d}^{\nu}:=\left(\vec{u}^{\nu} \cdot \vec{\nabla}_{h}\right) \vec{u}^{\nu}, \quad \vec{f}_{1}^{v}:=\vec{f}-\vec{d}^{v}
$$

where $\vec{\nabla}_{h}$ is a finite difference discretization of $\vec{\nabla}$, so that (7.1) can be expressed as

$$
-R^{-1} \nabla^{2} \vec{u}+\vec{\nabla} p=\vec{f}_{1}^{\nu}, \quad \vec{\nabla} \cdot \vec{u}=g .
$$

(3) The system (7.2) gives an equation for pressure $p$ which is

$$
Q_{h} p_{h}=h_{h}^{\nu}
$$

where the function $h_{h}^{\nu}$ is generated by $\vec{f}_{1}^{\nu}$ and $g_{h}$. Apply the PE method to (7.3), that is, do several conjugate iterations to update $p^{v+1}$ from $p^{\nu}$.

(4) Let

$$
\vec{f}_{2}^{v}=\vec{f}-\vec{\nabla} p^{\nu+1},
$$

then the first equation in (7.1) is the so-called convection diffusion equation

$$
-R^{-1} \nabla^{2} \vec{u}+(\vec{u} \cdot \vec{\nabla}) \vec{u}=\vec{f}_{2}^{v} .
$$

To update $\vec{u}^{v+1}$, solve (7.4) for $\vec{u}$. We discuss the solution procedure later. Go to step (2). 
For our numerical experiment, we used the Navier-Stokes equations of the form

$$
\begin{aligned}
-R^{-1} \nabla^{2} u+u u_{x}+v u_{y}+p_{x} & =f_{1}, \\
-R^{-1} \nabla^{2} v+u v_{x}+v v_{y}+p_{y} & =f_{2}, \\
u_{x}+v_{y} & =0,
\end{aligned}
$$

on $0<x, y<1$ where

$$
\begin{aligned}
& f_{1}=2 R^{-1} \pi^{2} \sin \pi x \sin \pi y+0.5 \pi \sin (2 \pi x)-\pi \sin \pi x \sin \pi y, \\
& f_{2}=2 R^{-1} \pi^{2} \cos \pi x \cos \pi y-0.5 \pi \sin (2 \pi y)+\pi \cos \pi x \cos \pi y .
\end{aligned}
$$

The values of $u$ and $v$ are specified on the boundary.

The exact solution is given by

$$
u=\sin \pi x \sin \pi y, \quad v=\cos \pi x \cos \pi y, \quad p=\cos \pi x \sin \pi y .
$$

Because of the nonlinearity of (7.4), the Full Approximation Scheme (FAS) was used for the multigrid solver. See [5] and [15] for a description of FAS. Moreover the full weighting was used in the fine-to-coarse transfers of both the solution and the residual functions. To employ a stable discretization, upwind differencing was used for the first derivatives in (7.4) when the mesh size $h$ was larger than $2 / R U$, where $U$ is the maximum value of $\vec{u}$ on a given domain, see [10]. Otherwise, central differencing was used to get the overall second-order accuracy. In [4], the authors mentioned that it is better to employ upwind differencing only in the relaxation sweeps, and central differencing in the residual transfers, but we obtained the best numerical solution when the same differencing was used in both relaxation sweeps and residual transfers. Also, the computation of $\vec{f}_{2}^{v}$ at coarser levels used upwind differencing.

Tables 5 and 6 show the error and accuracy of the solution when $R$ is 30 . Notice that the method is second-order accurate.

TABLE 5. Errors for $R=30$.

\begin{tabular}{c|cc}
\hline$N$ & $u$ & $p$ \\
\hline 32 & $7.34(-4)$ & $3.65(-4)$ \\
48 & $2.01(-4)$ & $1.55(-4)$ \\
64 & $9.35(-5)$ & $8.94(-5)$ \\
\hline
\end{tabular}

\section{Conclusion}

The PE method has been shown to be an efficient numerical method for solving the steady Stokes equations. Since the work is essentially proportional to the number 
TABLE 6. Accuracy of the solution $R=30$.

\begin{tabular}{c|cc}
\hline$N_{1}, N_{2}$ & $u$ & $p$ \\
\hline 64,48 & 2.7 & 1.9 \\
64,32 & 3.0 & 2.0 \\
48,32 & 3.2 & 2.1 \\
\hline
\end{tabular}

of grid points, the efficiency of this method is exceptional. We have also shown that the method advocated by Bramble and Pasciak [3] is not as efficient for the finite difference schemes used here.

The inf-sup condition plays an important role in obtaining the theoretical convergence rate of the PE method. Research is being carried out to obtain inf-sup conditions for some finite difference approximations of the Stokes equations with isotropic grids. Our work is not yet finished for anisotropic grids. However, we expect that the convergence rate for anisotropic grids would be as fast as that of isotropic grids.

The PE method has been extended to the Navier-Stokes equations for low Reynolds numbers. Research is continuing on improving this method. Work is also being carried out on applying the method to time-dependent problems and using the method with domain decomposition.

\section{Acknowledgements}

The work of the first author was supported by the TGRC-KOSEF. The U. S. Army Research Office under grants DAAL03-87-K-0028 and DAAL03-91-G-0094 supported the work of the second author.

\section{References}

[1] K. Arrow, L. Hurwitz and H. Uwaza, Studies in Nonlinear Programming (Stanford University Press, Stanford, 1958).

[2] A. K. Aziz and I. Babuska, "Survey lectures on the mathematical foundations of the finite element method", in The Mathematical Foundations of the Finite Element Method with Applications to Partial Differential Equations (ed. A. K. Aziz), (Academic Press, New York, 1972) 1-362.

[3] J. H. Bramble and J. E. Pasciak, "A preconditioning technique for indefinite systems resulting from mixed approximations of elliptic problems", Math. Comp. 50 (1988) 1-18.

[4] A. Brandt and N. Dinar, "Multigrid solutions to elliptic flow problems", in Numerical Methods for Partial Differential Equations (ed. S. V. Parter), (Academic Press, New York, 1979) 53-148.

[5] A. Brandt, "Guide to multigrid development", in Multigrid methods (eds. W. Hackbusch and U. Trottenberg), (Springer, New York, 1981) 220-312. 
[6] W. L. Briggs, Multigrid Tutorial (Lancaster Press, Lancaster, Pennsylvania, 1987).

[7] M. Crozier, Approximation et méthodes iteratives de resolution d' inequations variationnelles et de problèmes non linéares, IRIA cahier no. 12 (1974).

[8] M. Fortin and R. Glowinski, Resolution Numérique de Problèmes aux Limites par des Méthodes de Langrangien Augment (1981).

[9] V. Girault and P. A. Raviart, "Finite Element Approximation of the Navier-Stokes Equations", in: Lecture Notes in Mathematics 749 (Springer, New York, 1986).

[10] W. Hackbusch, Multi-Grid Methods and Applications (Springer, New York, 1985).

[11] J. C. Strikwerda, "Finite difference methods for the Stokes and Navier-Stokes equations", SIAM J. Sci. Stat. Comput. 5 (1984) 56-68.

[12] J. C. Strikwerda, "An iterative method for solving finite difference approximations to the Stokes equations", SIAM J. Numer. Anal. 21 (1984) 447-458.

[13] J. C. Strikwerda, Finite Difference Schemes and Partial Differential Equations (Wadsworth and Books/Cole, Pacific Grove, CA, 1989).

[14] J. C. Strikwerda and C. D. Scarbnick, "A domain decomposition method for incompressible viscous flow”, SIAM J. Sci. Stat. Comput. 14 (1993) 49-67.

[15] P. Wesseling, An Introduction To Multigrid Methods (John Wiley and Sons, New York, 1992). 